DOI: $10.17148 /$ IARJSET.2021.8946

\title{
IoT based Smart Face-Mask Dispensing Machine for Public
}

\author{
Priya J \\ Research Scholar, Bannari Amman Institute of Technology, Sathy, TamilNadu
}

\begin{abstract}
The COVID-19 pandemic has set the public eye on researchers, scientists and doctors who are working meticulously care for Covid patients, research on medicines and to develop vaccine for the virus and formulate health and safety conventions. Along with these people, innovative engineers who are being a part of the cause by inventing several interesting designs and products to make the combat the virus easier and to treat people safer. The usage of masks is very much essential for everyone nowadays to protect ourselves. The timely availability is much important. In schools and colleges or in public places like railway stations, airports, bus stations while travelling people might find it difficult to find the stores or shops selling to get a mask. Hence it is necessary to have a vending machine for easy access and availability at all times. This paper discusses the concept of an automated smart vending machine. The flexible Internet of Things (IoT) platform involves high customizability with a good Prognostics \& Health Management media. An implementation of the framework is worked upon, discussing the design of a mask-dispensing unit that can be used in schools, colleges and other public places of medium to a large gathering. The mask can be dispensed without any human intervention at the same time as maintaining high levels of well-being and reducing the cause of virus spread.
\end{abstract}

Keywords: COVID-19, Smart Dispensing Machine, Mask, IoT

\section{INTRODUCTION:}

The coronavirus COVID-19 was believed to be come from Wuhan, China during December, 2019. The novel virus is promptly spreading to more than 200 countries, and has turn out to be a pandemic. The unique coronavirus COVID-19 is spread predominantly by droplets from coughs, sneezes, sings or speaks of an infected person. Therefore appropriate use of face-mask is able to reduce infection by preventing droplets from spreading into the surroundings. There are various varieties of face-masks such as ready-made from stores, and home-made masks. They are made up of cloth or other materials. Actually cloth based face-mask is not an authentic respiratory protective device and may not be provide effective protection for the person who wearing it. Face-mask effectiveness is fairly dependent on as many people as possible wearing masks appropriately. According to the examination, the virus is spread at ease in crowded areas, indoor spaces wherever nearby contact with others cannot be evaded. It is safe to keep in mind that someone infected by COVID-19 coronavirus either may not be showing any symptoms or minor symptoms because of that the person is not mindful of being diseased. The virus can be multiply by this time a few days before any symptoms emerge out.

Our Government endorses the use of face-masks in every parts of the country. Further detailed recommendations on the use of face-masks in diverse situations hang on on the phases of the epidemic, and these can differ from one zone to another. People are out there doubt in a state of fear, anxiety and helplessness. The most recent suggestion indicates that face-mask aids prevent the transmission of coronaviruses and influenza viruses from symptomatic as well as asymptotic individuals and therefore, many more health care experts have acclaimed the use of face-mask everywhere for self-protection and to stay from spreading. The state government is duty-bound to educate the general public to tell between between good-quality masks with those fake face-masks via advertisement from social media. Weak subpopulation would also have intensified awareness to forged face-masks to avoid falling into the business scammers' mesh [1].

\section{IMPORTANCE OF FACE-MASK}

The World Health Organization (WHO) advises people about the usage of face-masks as part of a widespread solution of prevention and safety measure to limit the continuous transmission of COVID-19 coronavirus. Even a proper wearing of face-mask with social distancing provides adequate protection for a person. In addition to it, maintaining a frequent proper hand sanitizing and physical distancing while being in contact with a person, self-quarantine, periodical testing and adequate isolation with a appropriate ventilation in indoor sets and contact tracing with the help of various mobile applications introduced by government are comes under IPC (Infection Prevention and Control) measures. 
Though with all these control measures, it is critical to prevent human-to-human transmission of coronavirus. WHO continues to advise that anyone suspected or confirmed of having COVID-19 or awaiting viral laboratory test results should wear a medical mask when in the presence of others and this does not relate to those expecting a test earlier to travel. (Mask use in the context of COVID-19)

The usage of face-masks is endorsed in the following circumstances and for the following publics:

- People who are going be tested for the Covid-19 coronavirus, and afore the outcomes of the test are obtainable, if there is a critical requirement to be outdoor.

- While travelling in any mode of transport.

- $\quad$ Nomads arriving in a country from high-risk zones as they travel from their entrance to a place of isolation or if they have a vital reason to move outdoor during the isolation period.

It is worth and safe to wear a face-mask in circumstances where evading close contact is really tough. Regional experts may issue their own corresponding commendations for their zones founded on their own risk valuation [2].

As coronavirus blowout all across the world, the importance and the need of face-mask has been known to most of us. By now, only predominant solution is these tissues (masks) as being a barrier between the deadly virus and us, though it causes slight inconvenience during any workout. And this is urged by all healthcare officials includes WHO, CDC and local institutions from around the world. When we look back to the essentials, the primary role of face-masks is to prevent the transmission of coronavirus through respiratory droplets, which can easily pass in the mouth and nose of other person. On the other hand, if you wear the face-mask in a floppy way that won't help it out, it doesn't cover the mouth and nasal properly, and then the risk factor of getting the virus will definitely increase. In fact, the CDC has announced various statements and how-to info-graphics to accentuate the prominence of appropriately wearing face masks, requesting people to:

- Wear the face-mask over the mouth and nose properly, then fix firmly under the chin.

- $\quad$ Try to wear it tightly alongside of the face.

One foremost benefit of using face-masks is to safeguard the people nearby you, particularly if a person has been working in a crowded environment such as restaurants, grocery store etc., or suppose he/she exposed to someone act as a carrier of the coronavirus. Due to these many analyses, most of the governments and healthcare workers around the world urge people to safeguard themselves by following all the mandatory announcements made by them [3]. By the way further spreading of the virus can be stopped to an extent.

\section{COMPARATIVE ANALYSIS ON USAGE OF MASK}

The below given graphical analysis report Fig.1 shows that the variations in the number of Covid-19 coronavirus victims before and after the usage of face-masks. The comparative study is done based on the phase-I and phase-II Covid-19 patients count. The graph shows clearly that how face-masks really help-out during this pandemic. Here, random five countries were taken to the account. Based upon the WHO report, count has been considered. Consider the country Spain, where $92 \%$ of people among the whole population wearing face-mask properly which results in very less in number of Covid-19 cases. Whereas in India, though $75 \%$ of people among the total population using facemasks but mostly in floppy manner leads to rapid increase in number of Covid-19 cases.

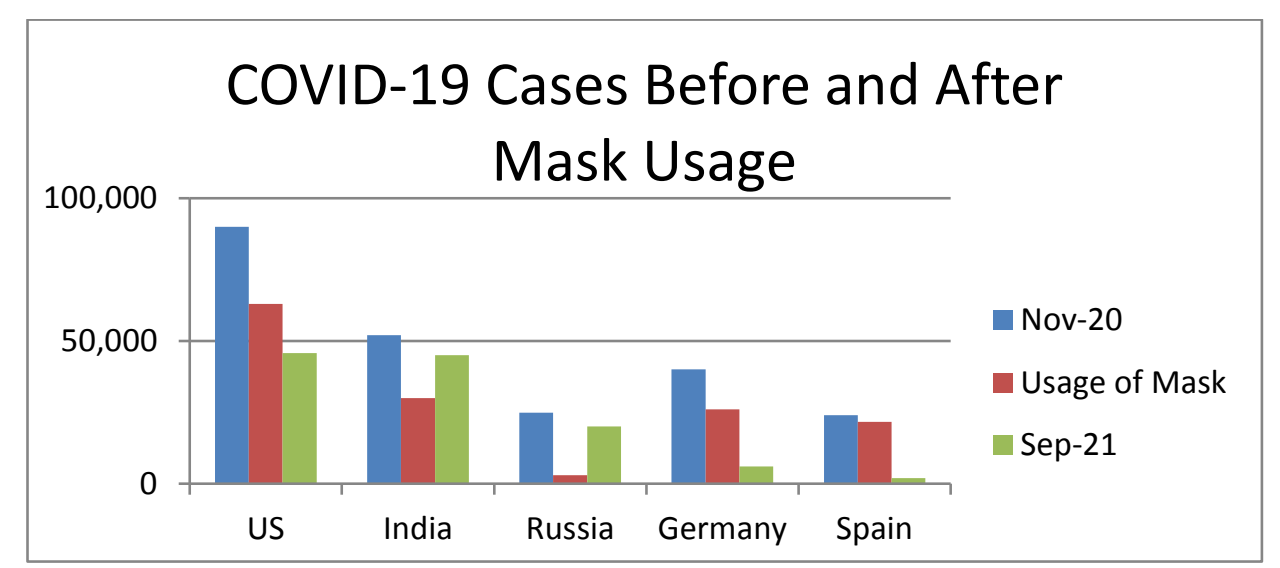

Fig.1. Comparative Analysis 
DOI: 10.17148/IARJSET.2021.8946

\section{PROPOSED MODEL}

There have been cases where people getting disappointed due to out of stock in vending machines. Frequent unavailability of stock in the machine makes people to neglect such type of machines and it would not suit for long run. Therefore, our proposed model of dispensing machine has also a unit for reporting non-availability of stock immediately to the reporting person and instantly the machine get refilled on time. The driving aspects of global facemask market are, (a) rising force on using face-masks everywhere, (b) increase in Covid-19 cases and (c) awareness formed by various organizations and healthcare workers.

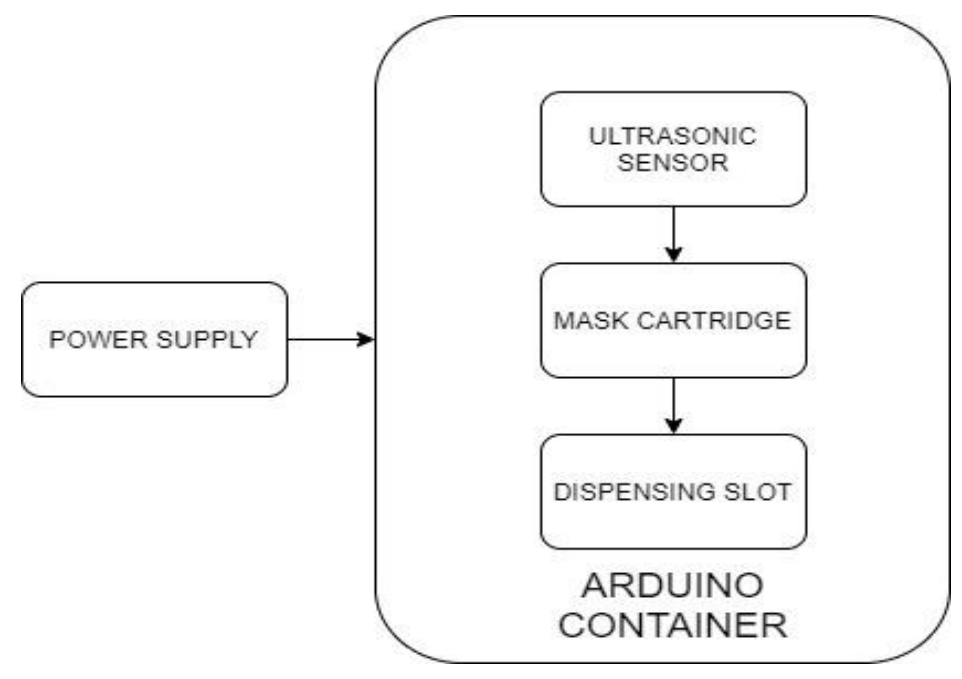

Fig.2. Block Diagram

The block diagram of the proposed model is given in the Fig.2. Basic needs for a mask dispensing machine includes, mask cartridge, dispensing slot and a container to hold these components with a power supply to make this dispensing automatic. Here, ultrasonic sensor is an add-on to maintain the un-touchability condition in order to prevent the spread of coronavirus from person to person.

To make the machine smart and automatic, the technology Internet of Things (IoT) is been used here. Smart technology, IoT comprised of sensors and actuators. Here ultrasonic sensor is used as input to the dispensing machine, which passes the user request to the cartridge. The mask cartridge works with the help of servo motors on each end of the roller. It helps to bring the face-mask to the dispensing slot from the original storage. Hence, when the power supply is on, the machine has to check for the availability of face-masks. Then if a person waves the hand in front of the ultrasonic sensor, a mask will come to the dispensing slot, where it can be collected.

\section{HARDWARE MODEL}

The hardware model of proposed system is given in the Fig. 3 shows the hardware connection between IoT devices which comprises of Arduino board plays the vital role as a container which holds all other sensors and actuators. Ultrasonic sensor to sense human interruption and to dispense the face-mask, two number of servo motors to bring up the face-mask from the container, led lights to show the availability of stock inside the cartridge. Power supply heads for all these actions. 


\section{International Advanced Research Journal in Science, Engineering and Technology}

Vol. 8, Issue 9, September 2021

DOI: $10.17148 /$ IARJSET.2021.8946

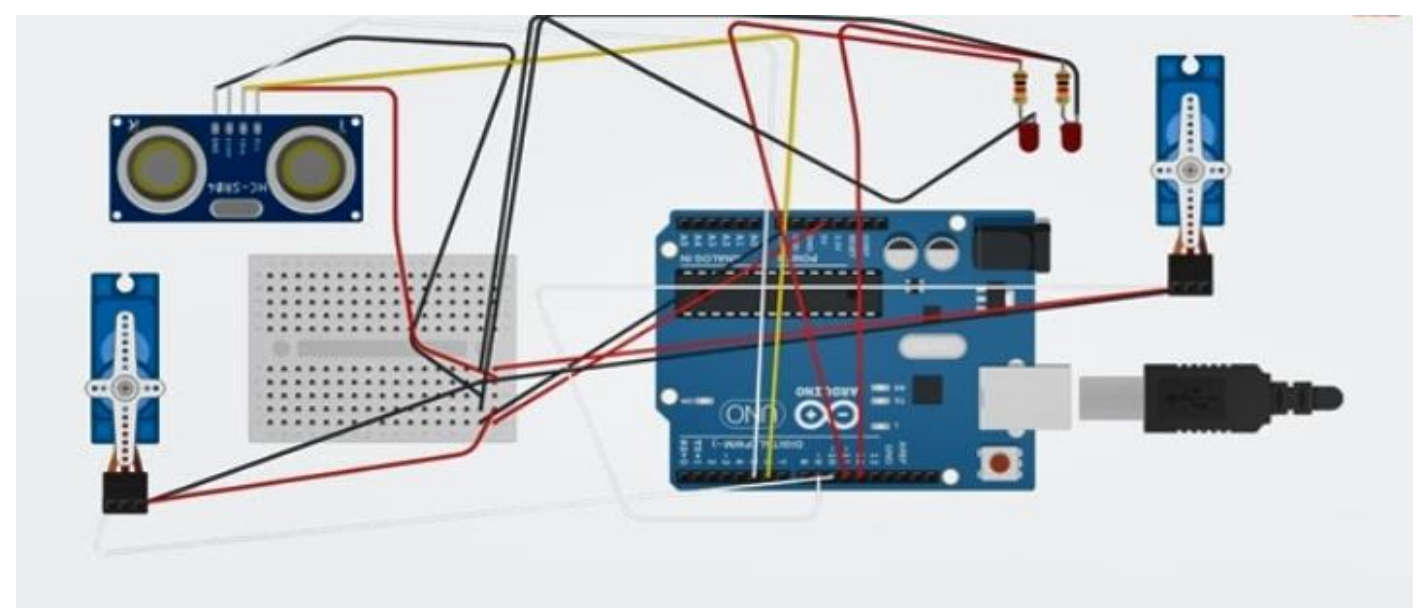

Fig.3. Hardware Model

\section{RESULTS AND DISCUSSION}

Working model of the proposed system is shown in the Fig.4, ultrasonic sensor has been placed in the right top corner where people use to wave hands by that they can collect the face-mask at the dispensing slot as per the procedure explained afore with all those components covered inside the dispensing machine.

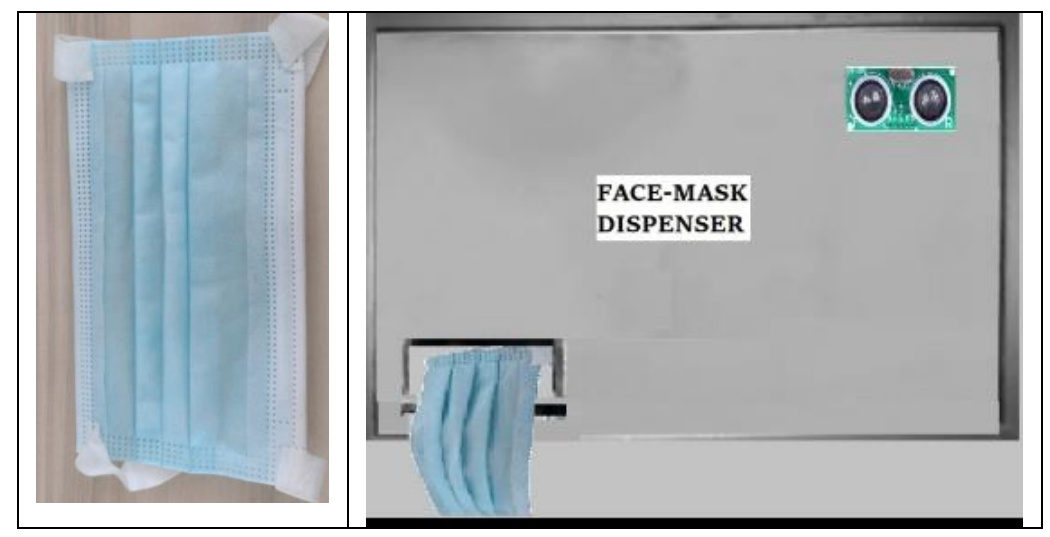

Fig.4. Working Model

During this pandemic situation, Covid-19 cases are massively rising; one of the first precautionary measures advised to people is to wear a mask. The electronic product development laboratory has come up with a QR-based mask vending machine", which is named MASKBOX. The first machine has been installed at electronic product development laboratory. The dispensary system works based on a motor-operated rotary dispensing mechanism. Hence, only small space would be required to store the surgical masks, and the restoration process takes very little time that amounts to almost zero downtime. The QR-based payment process makes a very quick response from the bank server that helps to immediately dispense the mask. Mask Box will be more useful at public places like railway stations, airports, metro rail stations, markets, theatres, and religious places. Employing this box in these public places may aid users get a mask even though the people forget to carry it.

\section{REFERENCES:}

1. Lam, Simon Ching; Suen, Lorna Kwai Ping; Cheung, Teris Cheuk Chi, "Global risk to the community and clinical setting: Flocking of fake masks and protective gears", May -2020.

2. Finnish Institute for Health and Welfare "Recommendation on the use of face masks for citizens", Retrieved 17 August 2020

3. Health \& Wellness, Patient safety /av World Medical Card, "The Importance of Wearing Masks", 25. August 2020. 